\title{
A serious games platform for validating sonification of human full-body movement qualities
}

\author{
Ksenia Kolykhalova \\ University of Genoa, Italy - \\ DIBRIS \\ ksenia.kolykhalova@ \\ dibris.unige.it
}

\author{
Paolo Alborno \\ University of Genoa, Italy - \\ DIBRIS \\ paolo.alborno@dibris.unige.it \\ Gualtiero Volpe \\ University of Genoa, Italy - \\ DIBRIS \\ gualtiero.volpe@unige.it
}

\author{
Antonio Camurri \\ University of Genoa, Italy - \\ DIBRIS \\ antonio.camurri@unige.it
}

\begin{abstract}
In this paper we describe a serious games platfrom for validating sonification of human full-body movement qualities. This platform supports the design and development of serious games aiming at validating (i) our techniques to measure expressive movement qualities, and (ii) the mapping strategies to translate such qualities in the auditory domain, by means of interactive sonification and active music experience. The platform is a part of a more general framework developed in the context of the EU ICT H2020 DANCE "Dancing in the dark" Project n.645553 that aims at enabling the perception of nonverbal artistic whole-body experiences to visual impaired people.
\end{abstract}

\section{Author Keywords}

Serious game; movement qualities; sonification; movement analysis

\section{ACM Classification Keywords \\ H.5.2. User Interface}

\section{INTRODUCTION}

In this paper, serious games are a tool to support the design, the development and validation of (i) novel techniques to measure expressive movement qualities, and of (ii) the mapping strategies to translate such qualities in the auditory domain, by means of interactive sonification and active music experience.

The work is carried out within the EU ICT H2020 DANCE "Dancing in the dark" Project n.645553 By integrating multidisciplinary research - neuroscience, affective computing,

Permission to make digital or hard copies of all or part of this work for personal or classroom use is granted without fee provided that copies are not made or distributed for profit or commercial advantage and that copies bear this notice and the full citation on the first page. Copyrights for components of this work owned by others than ACM must be honored. Abstracting with credit is permitted. To copy otherwise, or republish, to post on servers or to redistribute to lists, requires prior specific permission and/or a fee. Request permissions from permissions@ acm.org. (c) 2015 ACM ISBN/14/04 $\$ 15.00$

DOI string from ACM form confirmation nonverbal emotion and social signal processing, humancomputer interaction, sound and music computing, humanistic and artistic theories - the project is aiming at developing techniques and models for human body movement quality analysis, with a focus on the expressive component of nonverbal communication and on mapping of such qualities onto sound and music generation and processing.

We describe a platform to configure a specific kind of serious games related to the above described context i.e., for validating mappings between human movement qualities and parameters of sound models.

\section{Movement Qualities}

Full-body movements can be performed with different expressive qualities (e.g. rigidity, fluidity, impulsivity). With the term "movement quality" we mean the qualitative characteristics of the human body movements that are independent from the specific kind of movement which is performed. In previous studies it has been experimentally shown that movement qualities may communicate social relations and intentions, such as: emotional states [2], affiliation [8], cultural background [12], dominance [13], agreement [1], group cohesion [6], empathy [13].

In our study we use a multilayered framework approach to distinguish between different movement qualities and their measurements, i.e. movement features. In such framework low-level features (e.g., single joint velocity) are used to compute high level features (such as user intentions, emotions etc.). Analysis of expressive movement qualities is based on the recent studies in emotion recognition from body gestures by Piana et al. [11], on impulsivity and smoothness by Mazzarino and Mancini in [9] and by Niewiadomski et al. [10].

\section{Interactive Sonifications}

Sonification consists in the use of non-speech audio to convey information or perceptualize data [7]. Auditory perception takes advantages in temporal, spatial, amplitude, and frequency resolution that open possibilities as an alternative or complement to visualization techniques. 
Interactive sonification and musicalization of choreographic movements (processing of sound spaces and ways of using affective music content in relation to qualities of gestures) addresses forms of sensory substitution: to see through listening.

Recently sonification was used as a scientific method for improving the understanding of complex information. As Hermann defines in [5]: sonification is "data-dependent generation of sound, if the transformation is systematic, objective and reproducible, so that it can be used as scientific method". Dubus and Bresin [3] provided a systematic review on sonification in different research fields.

Institute for Research and Coordination Acoustic/Music (IRCAM) has explored numerous applications such as rehabilitation, sensory substitution, and movement learning in sport which can potentially benefit of interactive auditory feedback. For example they studied sensori-motor learning in gesturesound interactive systems (Legos Project ${ }^{1}$ ) and continued such research with an ongoing project about enhancing motion interaction through music performance (MIM Project ${ }^{2}$ ).

The remaining part of this paper is structured as follows: first we state the objectives and describe the general concept that lays behind our platform. Then, we introduce the architecture of the platform, explaining in details the component modules and the software implementation. Finally, we present an initial prototype of a serious game developed on the platform, named Move in the Dark.

\section{CONCEPT}

In the general framework of the DANCE Project we aim at mapping human movement qualities into sound by associating appropriate interactive sonifications to the extracted combinations of movement features in order to permit the perception of nonverbal artistic whole-body experiences to visual impaired people. Therefore, the main purpose of the proposed platform can be summarized as follows: to flexibly configure serious games able to teach users how "to hear" a dance (i.e. to understand movement qualities in terms of interactive sonifications) and, at the same time, to use these serious games as an efficient and useful tool for validation and comparison between different mappings of interactive sonifications with qualities of movements.

The proposed platform is a contribution to one of the research objectives of the EU DANCE Project, in particular for identifying effective mappings between physical gestures and sound parameters in order to support the development of feedback techniques and understand how to convey body motion through sound. Testing new perceptual experiences of body movement implies the possibility of rapprochement and sharing (of spaces and emotions) between visually impaired and sighted people.

The use of a game-based paradigm and concept to analyze users perception, allows us to set up an ecologic and natural environment for exploration of movements through sounds.

\footnotetext{
${ }^{1}$ http://legos.ircam.fr/

${ }^{2}$ http://mim.ircam.fr/
}

The prototypical serious game that the platform supports is described as follows. The game is a competition between two or more users. Users are blindfolded to increase the sensitivity of each user on the auditory channel, and to avoid imitation of other players. They listen to a selected sonification of one or more movement qualities, and are asked to move in such a way to match the qualities they perceive in the sound they are listening to. The same movements qualities, that originated the sonification, are measured from each user and compared with the ones produced by the system. Each player receives points according to the distance (by means of a given metrics) of her movement quality with respect to the qualities corresponding to the sonification. The player that best approximates the movement qualities of the listened sonification wins. This game concept represents a useful tool for investigating the perception of movement qualities when mapped into sounds.

The platform enables a flexible configuration of such a game by supporting the selection of sound models, expressive movement qualities and type of interaction between users (competition, collaboration etc.) in a particular game session. The platform consists of modules, that can be adjusted according to the needs and represents an open, expandable repository for additional interactive sonifications models and movement qualities.

Moreover, regarding to the supported types of interactions between users, we are not limiting it only to a competitive challenge. Rather we extend it to collaborative games and to cases where the rules of the game require cohesion, entrainment and emergence of leadership in a group of users. For example in the case of a collaborative game, a group of users can be asked to move as a whole to express the listened qualities.

\section{ARCHITECTURE}

The platform consists of a set of internally connected modules. Its architecture is shown in Figure 1. The platform is scalable: it can support onbody accelerometers and RGB-D sensors but it also can be extended to experiments with motion capture and multimodal sensor systems in a lab setup.

\section{Offline configuration}

Before starting an on line session, the platform needs to know which features are considered and how to sonify them and how to compute the user's scores. The platform configuration tool (see Figure 1) allows to select the followings parameters, and bind them together in tuples:

- Movement Feature Extraction Algorithm (MF-EA): All the feature extraction algorithms that are available in the platform by default, have been included in the DANCE Project software library. Each feature is computed from motion data, and some parameter can be specified (e.g., the id of the movement segment data selected from a movement segment repository). Examples of movement features are: lightness, heaviness, rigidness, fluidity, impulsivity, suddenness, symmetry, energy. In another paper in preparation we describe in detail our multi-layered conceptual 


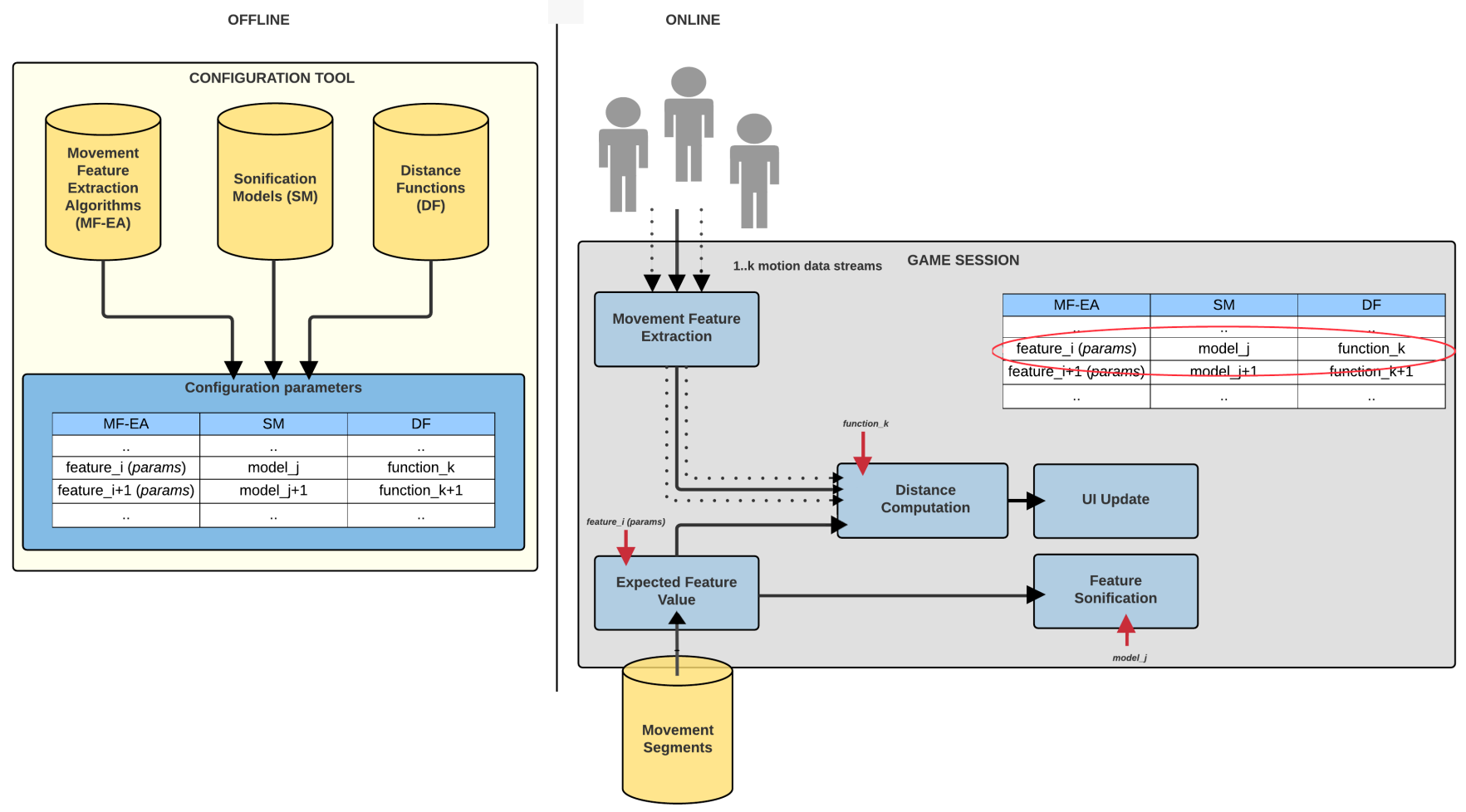

Figure 1. Platform Architecture

framework and the list of expressive features and qualities of movement at different levels of abstraction.

- Sonification Model (SM): Sonification models are used to "translate" a movement feature into sounds in real-time. Examples of sonification models are: granular synthesis, physical models and so on. The currently available interactive sonifications, and the criteria and strategies for their design are described in a paper in preparation.

\section{- Distance function (DF):}

At the end of each game session the degree of precision of each user in expressing the sonified movement qualities is computed. The distance function is the metric used to compute the difference between the value of all the selected features, sonified by the system, and the ones extracted and measured on the user's movements. A basic example of a distance function is the euclidean distance, but the platform supports the definition of additional functions (weighted and squared euclidean distance, correlation distance, etc.)

\section{Online session}

First of all, a single tuple (MF-EA - SM - DF) from the table generated by the configuration tool is picked. This choice is based on a decision that the game designer made in the offline configuration step. For example the tuples can be selected randomly (e.g. for needs for randomization in scientific experiments and evaluations) or according to a defined sequence of features (palimpsest).

The selected feature and its parameters are sent to the Expected Feature Value module that computes the feature values on a pre-recorded movement segment, stored in an annotated archive, identified by one of the parameters. Computed values are sent to the Feature Sonification module, that is responsible for sonifying them, for a certain time interval chosen in the configuration tool, before switching to the next tuple.

While users are moving, data streams are sent from the accelerometers to the platform. Acquired streams, are passed to the Movement Feature Extraction module that computes the value of each feature on the received data. At every time instant the distance between the feature extracted from the movements performed by the users and those received by the Expected Feature Value module is computed to provide a score. The score is computed by using the distance function selected in the configuration tool.

\section{System implementation}

The EyesWeb XMI open platform has been chosen to implement the platform ${ }^{3}$. EyesWeb is a development and prototyping software environment for both research purposes and interactive applications. It supports multimodal analysis, real-time processing of non verbal expressive gestures, research on synchronization, coordination, and entrainment in dance and music performance. The platform has been implemented in terms of a collection of software modules for EyesWeb. Extraction of features for analysis of motoric behavior has been implemented as software modules in the DANCE Software Library.

\footnotetext{
${ }^{3} h t t p: / / w w w . i n f o m u s . o r g / e y e s w e b_{i}$ ta.php
} 

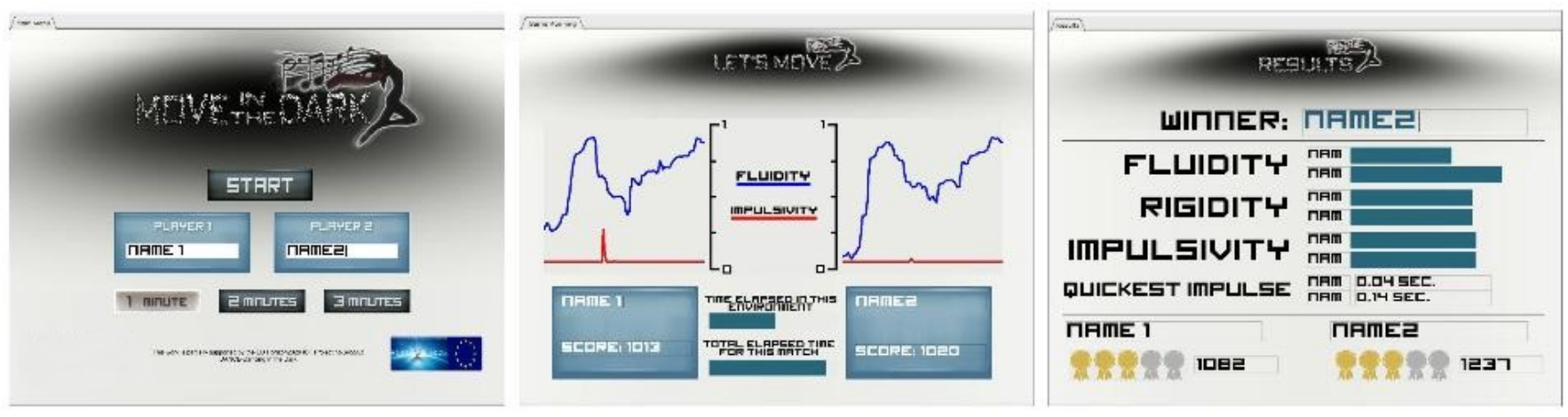

Figure 2. Game interface layouts

\section{INITIAL PROTOTYPE : MOVE IN THE DARK}

We developed an initial prototype named "Move in the dark" that presents a limited set of movement features to be sonified: energy, fluidity/rigidity and impulsivity and two common approaches to sound generation: granular synthesis and physical models. These features were selected based on studies and investigation of expressive full-body movements in the DANCE Project.

\section{Game session}

Each user is equipped with two smartphones ${ }^{4}$ attached to the arms with an armbands. The game (See a game session in Figure 3) is a two-player challenge following the rules described in the Concept Section, it consists of three phases:

Preparation: at the beginning of the game two players should write their names and how long they want to play $(1,2$ or 3 minutes).

Let's Move: the system starts to produce the first sonication of one among the available movement features (fluid, rigid and impulsive). Each player is asked to move with one of these movement features, i.e., with the quality that in her opinion matches to the sound she is listening to.

Check your scores: depending on how much each player has been able to correctly translate the sonifications into features, a winner is determined given the score. At the end of the session, the total scores, are visualized on the interface that is composed by three layouts, that refers to each game phase. The interface is shown in Figure 2).

\section{Sound model selection and evaluation}

In order to evaluate whether the selected sound models are suitable to be used in the game, we conducted a perceptive study with 16 participants. Participants explored how their movements were mapped into sound and got acquainted with the sonications. Then participants were asked to recognize the movement qualities only by listening to pre-recorded

\footnotetext{
${ }^{4}$ To extract information about the users motion, we used four Nexus $\mathrm{S}$ commercial Android smartphones. Each Nexus S are equipped with InvenSense MPU-6050 three-axis gyroscope and accelerometer.
}

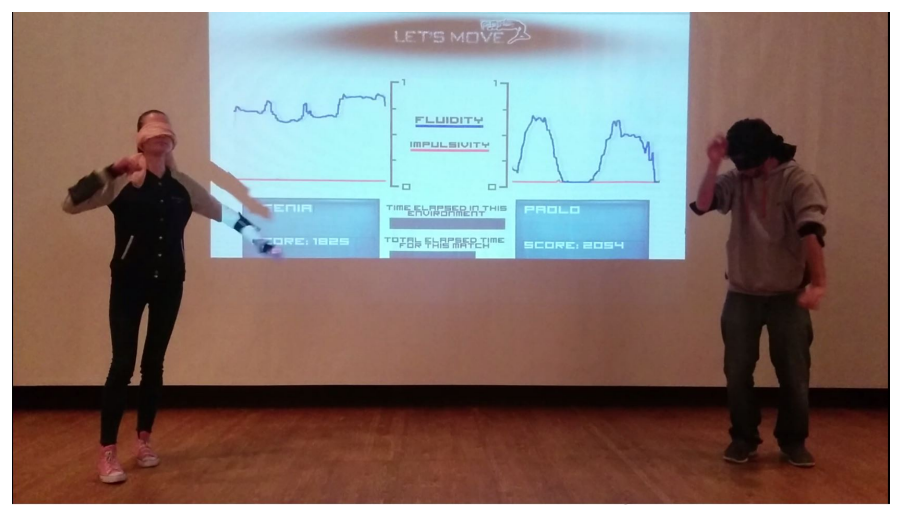

Figure 3. An instance of the platform at work

audio-files that were created using the same sound models. In this way, participants were in blind conditions and we investigated their capabilities and sensibilities in recognizing individual movement qualities only by means of the auditory modality. The group of participants included 16 people (13 $\mathrm{M}, 3 \mathrm{~F}$; diverse nationality; mean age 29 years, STD=5.67). We found that: sound was responsive to movement (mean 4 with STD 0.73) and easy to manipulate (mean 3.81 with STD 0.655). Participants stated that it was easy to manipulate the sound corresponding to the following movement features: fluidity (mean 3.63 with STD 0.957), energy (mean 4.31 with STD 0.793 ) and that they could distinguish between rigidity and impulsivity sonications (mean 3.63 with STD 1.147). According to these results we conclude that the chosen sound models can be used for Move in the dark.

In Table 1 the detailed information of the feedback from participants is provided.

\section{CONCLUSIONS AND FUTURE WORK}

Move in the Dark was presented at Mundaneum Museum in Mons, Belgium in the framework of the International Workshop Enterface 2015. After that the platform architecture was modified and improved according to the feedback of the users and presented at Festival della Scienza, Genova, Italy on 27 November 2015. 


\begin{tabular}{|c|c|c|c|c|}
\hline Question & Min & Max & Mean & STD \\
\hline $\begin{array}{c}\text { How responsive was the sound } \\
\text { to the actions you performed? }\end{array}$ & 3 & 5 & 4.00 & 0.730 \\
\hline How well could you manipulate the sounds? & 3 & 5 & 3.81 & 0.655 \\
\hline I thought the sounds were pleasing & 1 & 5 & 3.13 & 1.258 \\
\hline $\begin{array}{c}\text { It was easy for me to manipulate } \\
\text { the sounds related to fluidity }\end{array}$ & 2 & 5 & 3.63 & 0.957 \\
\hline $\begin{array}{c}\text { I had to put too much strength into } \\
\text { creating an impulse }\end{array}$ & 1 & 4 & 2.44 & 1.153 \\
\hline $\begin{array}{c}\text { It was easy for me to differentiate } \\
\text { rigid and impulsive movements }\end{array}$ & 2 & 5 & 3.63 & 1.147 \\
\hline $\begin{array}{c}\text { I could notice the difference between using one, } \\
\text { two or more limbs during the energy experiment }\end{array}$ & 1 & 5 & 2.88 & 1.310 \\
\hline
\end{tabular}

Table 1. Feedback about sonifications. (6-points Likert scale from 0 - not at all to 5 - very much)

In order to use the platform as a validation tool of the perception mapping between sonification models and expressive movement qualities in the future work we are going to test the platform with a number of users. They will try out different configurations of the game sessions. The sessions will be extended with other movement features and sound models (with respect to Move in the Dark). Then we want to conduct the perceptive studies in order to acquire the information about which sound models to which movement features corresponds better. In the framework of DANCE Project, the founded good sound models after that can be used for translation the expressive movement qualities into sound for visualimpared and normal sighted people.

\section{REFERENCES}

1. K. Bousmalis, L. Morency, and M. Pantic. Modeling hidden dynamics of multimodal cues for spontaneous agreement and disagreement recognition. In Automatic Face Gesture Recognition and Workshops (FG 2011), 2011 IEEE International Conference on, pages 746-752, 2011.

2. K. L. C. G. Castellano, G. Multimodal emotion recognition from expressive faces, body gestures and speech. In Doctoral Consortium of ACII, Lisbon, 2007.

3. B. R. Dubus, G. A systematic review of mapping strategies for the sonification of physical quantities. Plos One, 8(12), 2013.

4. K. Felix Navarro, E. Lawrence, J. Garcia Marin, and C. Sax. A dynamic and customisable layered serious game design framework for improving the physical and mental health of the aged and the infirm. In Conference on eHealth, Telemedicine, and Social Medicine. IARIA Conference, 2011.

5. T. Hermann. Taxonomy and Definitions for Sonification and Auditory Display. In P. Susini and O. Warusfel, editors, Proceedings of the 14th International
Conference on Auditory Display (ICAD 2008). IRCAM, 2008.

6. H. Hung and D. Gatica-Perez. Estimating cohesion in small groups using audio-visual nonverbal behavior. Multimedia, IEEE Transactions on, 12(6):563-575, 2010.

7. G. Kramer. Auditory Display: Sonification, Audification, and Auditory Interfaces. Perseus Publishing, 1993.

8. D. Lakens and M. Stel. If they move in sync, they must feel in sync: Movement synchrony leads to attribution of rapport and entitativity. Social Congnition, 29 (1):1-14, 2011.

9. B. Mazzarino and M. Mancini. The need for impulsivity and smoothness improving hci by qualitatively measuring new high-level human motion features. International Conference on Signal Processing and Multimedia Applications, pages 62-67, 2009.

10. R. Niewiadomski, M. Mancini, G. Volpe, and A. Camurri. Automated detection of impulsive movements in hci. In Proceedings of the 11th Biannual Conference on Italian SIGCHI Chapter, CHItaly 2015, pages 166-169, New York, NY, USA, 2015. ACM.

11. S. A. C. A. O. F. H. J. Piana, S. Adaptive body gesture representation for automatic emotion recognition. In Transactions on Interactive Intelligent System, 2015.

12. M. Rehm. Non-symbolic gestural interaction for aml. In D. R.-C. A. J. Aghajan, H., editor, Human-Centric: Interface for Ambient Intelligence, pages 327-345. ACM Press, Amsterdam, The Netherlands, 2010.

13. G. Varni, A. Camurri, P. Coletta, and G. Volpe. Toward a real-time automated measure of empathy and dominance. In Computational Science and Engineering, 2009. CSE '09. International Conference on, volume 4, pages 843-848, 2009. 